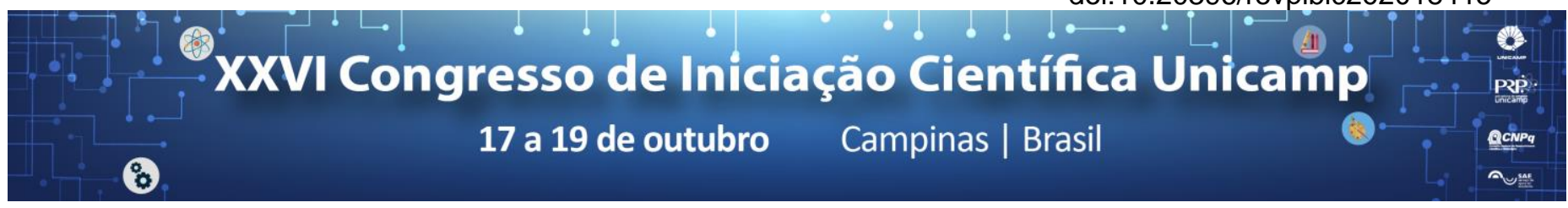

\title{
Generation of stochastic signals for dynamic structural analysis
}

\author{
Felipe M. Aranha, Gustavo H. Siqueira
}

\begin{abstract}
This paper aims to obtain a method capable of generating a random signal that has a specific Power Density Function and a predefined Power Density Function. Modeling these signals is essential for civil engineering purposes. Indeed, it is indispensable when we consider, for example, the reliability and the ergonomics of bridges and offshore platforms that are subjected to wind.
\end{abstract}

Key words: Signal generation, Power Density Function, Power Spectral Density.

\section{Introduction}

There is a great deal of mechanical problems that can be associated to the vibratory response of mechanical systems under random solicitations. Many of these solicitations have a non-Gaussian distribution. However, the models we possess nowadays end generating signals that end up following a Gaussian distribution. As a consequence, the model does not perfectly match the reality, what causes the construction of overpriced structures such as the Olympic stadium in São Paulo.

As a consequence, this paper is devoted to the development of a method capable of generating more realistic signals. In other words, signals that respect not only the real Probability Density Function, but also its Power Spectral Density.

To achieve this goal, we first develop a method that generates a Gaussian signal with pre-established Power Spectral Density. Then, we analyze a method that generates a random signal with desired Power Spectral Density and Laplace Probability Distribution.

\section{Results and Discussion}

We analyzed two different methods that are able to generate a Gaussian signal with predefined Power Spectral Density: the Random Spectral Phase [1] and the Source-Filter [2] Methods.

Both methods were tested with MATLAB and the results offered an acceptably close Power Spectral Density to the desired one. Even though the Source-Filter method seems less accurate, it has the advantage of not depending on the number of points we decide to use $(\mathrm{N})$, as opposed to the first one. For the Random Spectral Phase method, we have that the number of points $N$ determines the length of the time domain signal. Therefore, if we were to produce a longer lasting signal we would have to restart the program with an increased $\mathrm{N}$. On the other hand, the second method gives us a filter that can be used to generate a Gaussian signal as long as we want. Indeed, its advantage is that we can generate a signal in real time. In other words, we could actually build this filter, use a white noise generator as its input, turn it on and generate, at real time, the desired output signal.
Then, we were able to generate a random signal with desired Power Spectral Density and Laplace Probability Distribution using the Autoregressive Random Coefficients (ARRC) method. Indeed, Figure 1 demonstrates the result for the probability distribution that was obtained.

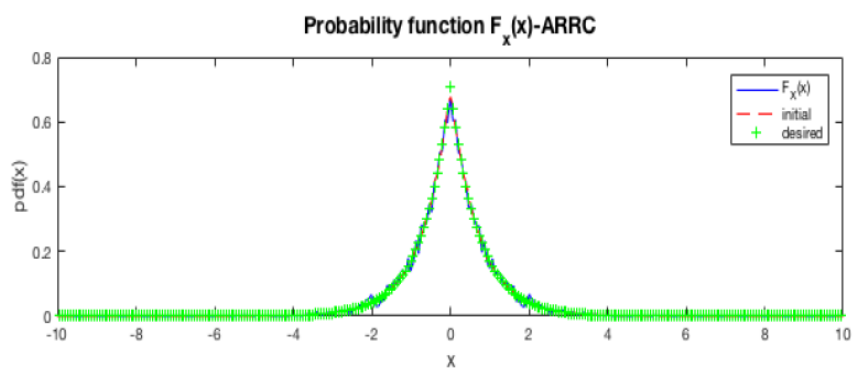

Figure 1: Verification of the Laplace Probability Distribution function obtained by the ARRC method.

\section{Conclusions}

This study allowed us to obtain three different methods when it comes to generating random signals. While the Random Spectral Phase and the Source-Filter Methods generate a Gaussian signal that has a predefined Power Spectral Density, the ARRC method generates signals that have different probabilities. It is important to note that not all probability distributions can be generated. Consequently, further research is necessary.

\section{Acknowledgement}

I thank PIBIC and CNPq for the opportunity. I also thank my supervisor, PhD Gustavo Henrique Siqueira for his help and patience.

\footnotetext{
${ }^{1}$ Christopher G. Fox. An inverse transform algorithm for generating random signals os a specified spectral form. Computers \& Geosciences, Vol 13: pp. 369-374, 1987.

${ }^{2}$ S. Plaszczynski. Generating long streams of $1 / \mathrm{f}^{\alpha}$ noise. In Fluctuations and Noise Letters, volume 0. World Scientific Publishing Company, 2006.
} 